\title{
ESTUDO COMPARATIVO DO TROFISMO DO MULTIFIDO NA ARTRODESE LOMBAR ABERTA VERSUS MINIMAMENTE INVASIVA
}

\author{
COMPARATIVE STUDY OF TROPISM OF THE MULTIFIDUS MUSCLE IN OPEN LUMBAR \\ ARTHRODESIS VERSUS MINIMALLY INVASIVE ARTHRODESIS

\section{ESTUDIO COMPARATIVO DE TROFISMO DEL MULTÍFIDO EN LA ARTRODESIS LUMBAR ABIERTA VERSUS LA MINIMAMENTE INVASIVA}

Cristiano Magalhaes Menezes ${ }^{1}$, Marcos Antonio Ferreira Júnior ${ }^{1}$, Roberto Sakamoto Falcon², Breno Frota Siqueira ${ }^{3}$

\section{RESUMO}

Objetivo: Determinar se a abordagem MIS para artrodese lombar em um nível reduz as alterações estruturais do multífido, tais como a diminuição de sua área de secção transversa e a degeneração gordurosa patológica, quando comparada a uma abordagem convencional aberta. Métodos: Entre Janeiro de 2007 e Janeiro de 2010, foram avaliados 27 pacientes submetidos a procedimento cirúrgico de artrodese aberta e MIS. Realizou-se RNM no pós-operatório, no intervalo entre 12 e 36 meses após a cirurgia, com vizualizacao do músculo multífido para seu estudo. Resultados: Todos os pacientes foram operados num nível de artrodese através da técnica aberta e MIS. Conclusão: Não foram encontradas diferenças significativas referentes às variáveis sexo e idade com a área e trofismo do multífido em ambos os lados.

Descritores: Artrodese; Vértebras lombares; Procedimentos cirúrgicos minimamente invasivos.

\begin{abstract}
Objective: Determine if the minimally invasive surgery (MIS) approach for lumbar fusion in a determined level can reduce structural changes in the multifidus muscle, such as decrease their cross-sectional area and pathologic fatty degeneration, compared to a conventional open approach. Methods: Between January 2007 and January 201027 patients undergoing surgical procedure and arthrodesis MIS were evaluated. Open MRI was performed in the postoperative period, between 12 and 36 months after surgery, with visualization of the multifidus muscle for its study. Results: All patients were operated on a level of arthrodesis using the open technique and MIS. Conclusion: No significant differences were found regarding the age and sex with the area and tropism of multifidus muscle on both sides.
\end{abstract}

Keywords: Arthrodesis; Lumbar vertebrae; Surgical procedures, minimally invasive.

\section{RESUMEN}

Objetivo: Determinar si un enfoque de MIS, para la artrodesis lumbar en un nivel, reduce los cambios estructurales en el multífido, como la reducción de su área de sección transversal y la patología de la degeneración grasa, en comparación con un enfoque abierto convencional. Métodos: Entre enero de 2007 y enero de 2010, se evaluaron 27 pacientes sometidos a procedimiento quirúrgico de artrodesis abierta y MIS. Se realizó RNM en el posoperatorio, entre 12 y 36 meses después de la cirugía, con la visualización del músculo multífido para su estudio. Resultados: Todos los pacientes fueron operados en un nivel de artrodesis con la técnica abierta y MIS. Conclusión: No se encontraron diferencias significativas respecto a las variables de sexo y edad, con el área y el trofismo del multífido en ambos lados.

Descriptores: Artrodesis; Vértebras lumbares; Procedimientos quirúrgicos mínimamente invasivos.

\section{INTRODUÇÃO}

As técnicas cirúrgicas para artrodese lombar sejam do tipo aberta ou, mais recentemente, minimamente invasiva, vêm sendo utilizadas com bons resultados para o manejo de uma variedade de problemas de coluna que necessitem de estabilização cirúrgica ${ }^{1}$.

Os músculos paravertebrais mais relevantes na sustentação da coluna lombar são: multífido, longuíssimo do dorso e íliocostal. O músculo multífido apresenta uma série de fascículos que se situam entre os processos espinhosos, lâminas e apófises transversas. Ele posiciona-se mais medial e posteriormente e está em íntimo contato com um grupo de músculos menores (interespinhais, rotadores curtos e intertransversos), sendo o principal estabilizador capaz de proporcionar a rotação posterior da vértebra no plano sagital ${ }^{2}$. O multífido é inervado somente pela porção medial do ramo posterior do nervo sinuvertebral, e não há suprimento nervoso intersegmentar, como ocorre em outros músculos do dorso ${ }^{3}$.

Os riscos associados à cirurgia aberta incluem maior risco de infecção, maior perda sanguínea, maior dano à musculatura paraespinhal, tempo intraoperatório e de internação mais prolongado, maior risco de lesão pelo instrumental utilizado e maior incidência de lesão neurológica associada ${ }^{1,4}$

O conceito de cirurgia minimamente invasiva não significa apenas incisões curtas, mas também menor lesão tecidual e resultado terapêutico máximo. A utilização da técnica de artrodese lombar minimamente invasiva tem como beneficio uma importante preservação da massa muscular paravertebral, bem como uma baixa morbidade em comparação à técnica cirúrgica convencional, aber-

1. Cirurgião de Coluna do Hospital Ortopédico /Lifecenter de Belo Horizonte (MG), Brasil.

2. Chefe do Servico de Cirurgia Espinhal do Hospital Ortopédico /Lifecenter de Belo Horizonte (MG), Brasil.

3. Residente do Servico de Cirurgia Espinhal do Hospital Ortopédico/Lifecenter de Belo Horizonte (MG), Brasil.

Trabalho realizado no Hospital Ortopédico /Lifecenter de Belo Horizonte (MG), Brasil.

Correspondência: QQSL11, área especial 1/17 -Taguatinga-Sul -Taguatinga. DF. Brasil. brenofrota_23@hotmail.com 
ta ${ }^{1,5}$. Existem evidências de que a desinserção e desvascularização da musculatura paravertebral podem levar à diminuição da força muscular do tronco, à instabilidade tardia da coluna vertebral e, potencialmente, à dor crônica na região lombar ${ }^{1}$.

A abordagem minimamente invasiva está associada a menores índices de pressão intramuscular, de edema paravertebral residual, de perda sanguínea e de produção de citocinas inflamatórias ${ }^{1}$.

Esse estudo tem como objetivo principal determinar se a abordagem minimamente invasiva para artrodese lombar em um nível reduz as alterações estruturais do multífido, tais como a diminuição de sua área de secção transversa e a degeneração gordurosa patológica, quando comparada a uma abordagem convencional aberta.

\section{MATERIAIS E MÉTODOS}

Entre Janeiro de 2007 a Janeiro de 2010, foram avaliados 27 pacientes submetidos a um procedimento cirúrgico de artrodese lombar em um nível. Destes, 14 foram submetidos à cirurgia convencional aberta (grupo A) e 13 foram submetidos a uma abordagem minimamente invasiva (grupo M). Todos os pacientes assinaram um termo de consentimento livre e esclarecido para a sua inclusão no estudo. Estudo analítico prospectivo analisando pacientes no período mencionado acima, num total de 27, que foram distribuídos de forma aleatória (randomização simples) em grupos para cirurgia convencional aberta (grupo A) e abordagem minimamente invasiva (grupo M).

As indicações cirúrgicas foram: doença degenerativa do disco associada ou não à hérnia discal ou à estenose do canal lombar e espondilolistese de baixo grau espondilolítica ou degenerativa. Todos os pacientes submeteram-se a um tratamento conservador mínimo de seis meses antes da cirurgia e realizaram exames pré-operatórios que incluíam radiografias estáticas e dinâmicas da coluna lombar e ressonância magnética (RNM). Os critérios de exclusão adotados pelos autores foram: 1) história de cirurgia lombar prévia; 2) cirurgia em mais de um nível.

A escolha da técnica cirúrgica para cada paciente seguiu um padrão aleatório. Adotou-se o protocolo habitual para avaliação da coluna lombar pós-cirúrgica. Dessa forma, todos os 27 pacientes realizaram RNM em diferentes períodos no pós-operatório, num intervalo entre 12, 24 e 36 meses e após a cirurgia. O aparelho utilizado para fazer a mensuração em cortes transversais e graduação para sinal em T2 foi de alta resolução com 1,5 tesla, permitindo, desse modo, a adequada visualização do músculo multífido. 0 volume muscular do múltifido foi avaliado quantitativamente por meio da área em mm, medida manualmente em cortes axiais ponderados em T1, paralelos ao interespaço vertebral, no nível dos pedículos de L3, L4, L5, S1 e S2. O grau de atrofia foi avaliado de forma qualitativa por meio da comparação entre a quantidade de tecido gorduroso e muscular seguindo a proposta de Goutallier para avaliação do trofismo do manguito rotador. Nesta classificação é possível quantificar o grau de degeneração gordurosa pontuando os achados encontrados na RNM de 0 a 4, conforme aumenta progressivamente a proporção gordura/músculo.

Os grupos A e M foram comparados em relação às seguintes variáveis: o nível da artrodese realizada, a área de corte transverso do multífido ao nível dos pedículos em cada lado da coluna, o grau de degeneração gordurosa e a presença de edema residual.

A análise estatística dos dados obtidos foi realizada com o programa SPSS, versão 10.0. Utilizou-se o Teste de Fisher e o teste t com diferenciação de média, considerando como significância o valor de $p<0,05$.

\section{Técnica para a MIS}

Após a indução anestésica geral o paciente é posicionado em decúbito ventral sobre um suporte de coluna radiotransparente, e realizada uma incisão de $2,5 \mathrm{~cm}$ de comprimento, a uma distância de $4,5 \mathrm{~cm}$ da linha média para acesso ao complexo facetário, conseguida através de dilatadores seqüenciais até a colocação de um tubo de 22 ou 26 mm de diâmetro com profundidade especifica para cada paciente 4 . Dissecção profunda limita-se à porção médio-lateral da lamina, faceta articular inferior, borda medial da faceta articular superior e istmo vertebral, mantendo a origem e inserção do músculo multífidus no processo espinhoso e borda lateral da faceta articular superior, respectivamente. Realizada facetectomia total com uso de broca de alta rotação com exposição da porção póstero-lateral do disco intervertebral. Exposição das raízes descendente e transversa pela ressecção do ligamento amarelo. Visualização do anulo discal, com anulotomia após cauterização dos vasos epidurais através da zona triangular de segurança e com adequada preparação das plataformas vertebrais com utilização de curetas, raspas e dilatadores específicos. Enxerto autólogo retirado da crista ilíaca obtido percutaneamente pela utilização de uma trefina preenche o espaço interssomático dentro e fora de um espaçador próprio. Artrodese posterior contralateral é realizada, o tubo retrator é retirado com cuidado e um possível sangramento muscular é controlado com uso do cautério bipolar. Uma vez que a fusão interssomática tenha sido concluída, realiza-se instrumentação pedicular através da mesma incisão com auxilio de agulha de Jamshidi e fio guia. Finalmente ocorre a introdução percutânea dos parafusos multiaxiais previamente medidos e das hastes. Todo o sistema é submetido à compressão para garantir estabilidade e recriar a lordose. Feridas cirúrgicas suturadas.

\section{Técnica aberta}

Incisão na linha media posterior na pele de aproximadamente $12 \mathrm{~cm}$ é feita. Musculatura paravertebral dissecada subperiostalmente, expondo os processos espinhosos e laminas. Instrumentações bilaterais com parafusos e hastes são realizadas. Facetectomia e laminectomia. As demais etapas foram as mesmas utilizadas para a MIS.

\section{RESULTADOS}

Os dados obtidos foram comparáveis entre os grupos, sendo a média de idade entre o grupo A para mulheres de 54,6 anos (variando entre 39 e 70 anos), que corresponde a 71,43\% (total de mulheres do grupo A), e para homens de 56,75 anos (variando entre 49 e 65 anos), correspondendo a 28,57\% (total de homens no grupo A). No grupo M, as mulheres apresentaram média de 63,8 anos (variando entre 31 e 83 anos), que corresponde a 38,46\% (total de mulheres do grupo M), e os homens com 45,5 anos (variando entre 32 e 62 anos), que corresponde a $61,54 \%$ (total de homens do grupo M). Havia quatro homens e dez mulheres no grupo A e oito homens e cinco mulheres no grupo $\mathrm{M}$. Todos os pacientes desse estudo foram operados em um nível de artrodese, sendo que, oito pacientes do grupo A foram no nível L4-L5 $(57,15 \%)$ e seis em L5-S1 (42,85\%). Já no grupo M, oito pacientes operados no nível L4-L5 $(61,54 \%)$ e cinco em L5-S1 (38,46\%). Edema residual foi observado em $57,14 \%$ no grupo A contra $23,07 \%$ no grupo $M$. Não foram encontrada diferenças significativas nos dados obtidos referente às correlações entre as variáveis sexo e idade com a área e trofismo do multífido em ambos os lados e nem com o tipo de cirurgia adotada, seja tipo aberto ou MIS.

A análise do trofismo do multífido foi realizada através da classificação de Goutallier. O resultado foi agrupado em degeneração não patológica (graus 0,1 e 2) e patológica (graus 3 e 4). No que se refere à avaliação entre o trofismo do multífido com o tipo de cirurgia, utilizando um nível significância de 5\% para $p<0,05$, pode-se observar que nos níveis L4,L5,S1,S2 à esquerda e nos níveis S1 e S2 à direita existem dependência quanto ao tipo de cirurgia (Figura 1).

Para descobrir qual o melhor tipo de cirurgia, foi utilizado a comparação de médias para mostrar aquela que apresentava os maiores níveis de trofismo. Essa comparação foi realizada em ambos os lados e observou-se que, em todos os níveis, o trofismo no grupo $\mathrm{M}$ foi menor $(\mathrm{p}<0,05)$ que o encontrado no grupo A bilateralmente (Figura 2). 
Por fim, a área do multífido de cada lado foi analisada e correlacionada com o tipo de cirurgia previamente realizada. Os resultados obtidos evidenciam a presença de maior atrofia muscular pós-operatória $(p<0,05)$ entre os pacientes do grupo A (Figura 3).

Não houve comparação prévia às cirurgias. Foram analisadas as variações do multífido e comparadas entre os dos grupos.

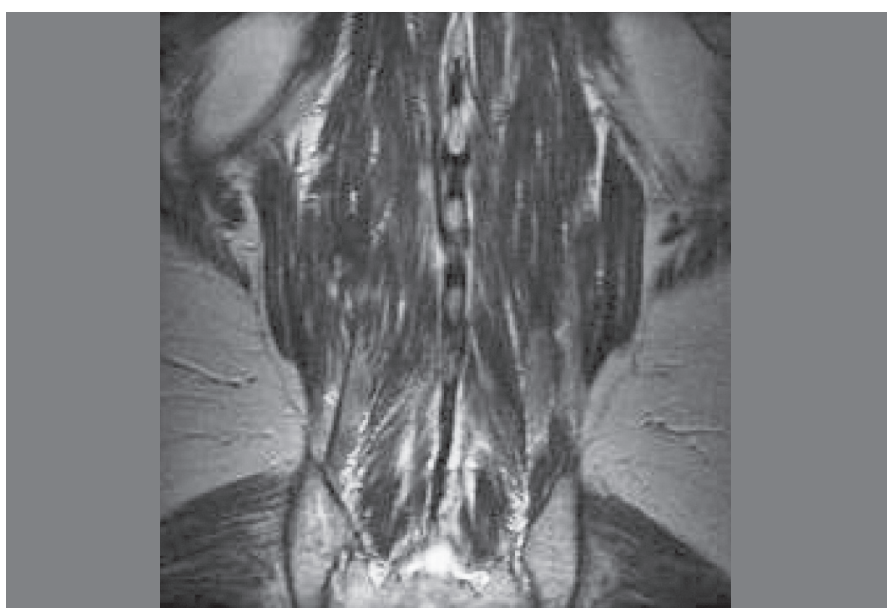

Figura 1. Trofismo do multífido na cirurgia aberta (12 meses de pós-operatório).

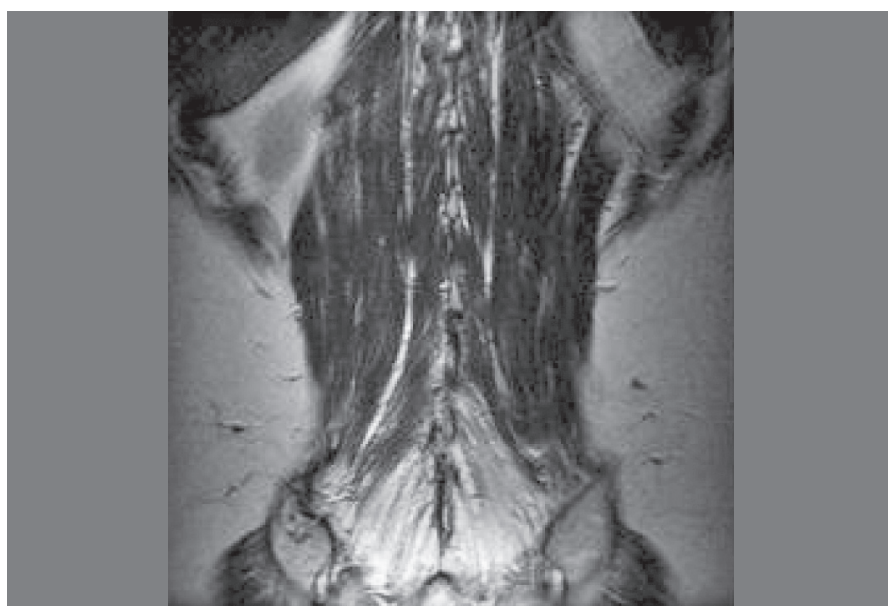

Figura 2. Trofismo do multífido na cirurgia minimamente invasiva (12 meses de pós-operatório).

\section{DISCUSSÃO}

Embora as vantagens biomecânicas e clínicas convencionais da cirurgia aberta para artrodese lombar sejam amplamente aceitas, a fusão espinhal com instrumentação aberta exige ampla dissecção muscular e de tecidos moles ${ }^{14}$. Esta dissecção, acompanhada pela desnervação das cápsulas das facetas e enfraquecimento de outras estruturas de apoio, dá origem a um efeito prolongado de menor recuperação funcional ideal ${ }^{6,7}$. Além disso, a fixação do parafuso pedicular aberto está associado com perda excessiva de sangue, maior taxa de infecção, bem como prolongamento do tempo operatório e de internação ${ }^{4}$.

Entre os fatores de riscos que aumentam a incidência de complicações e morbidades pós-operatórias estão a lesão direta, através da dissecção de áreas adjacentes à coluna e da retração com o instrumental cirúrgico no intraoperatório. O uso de afastadores durante a cirurgia por período maior que $2 \mathrm{~h}$ pode aumentar a pressão intramuscular e levar a alterações isquêmicas, causando anormalidades e atrofia dos músculos paraespinhais ${ }^{4,8}$.

A cirurgia minimamente invasiva da coluna foi desenvolvida com o objetivo de reduzir os graus de lesão muscular iatrogênica man-

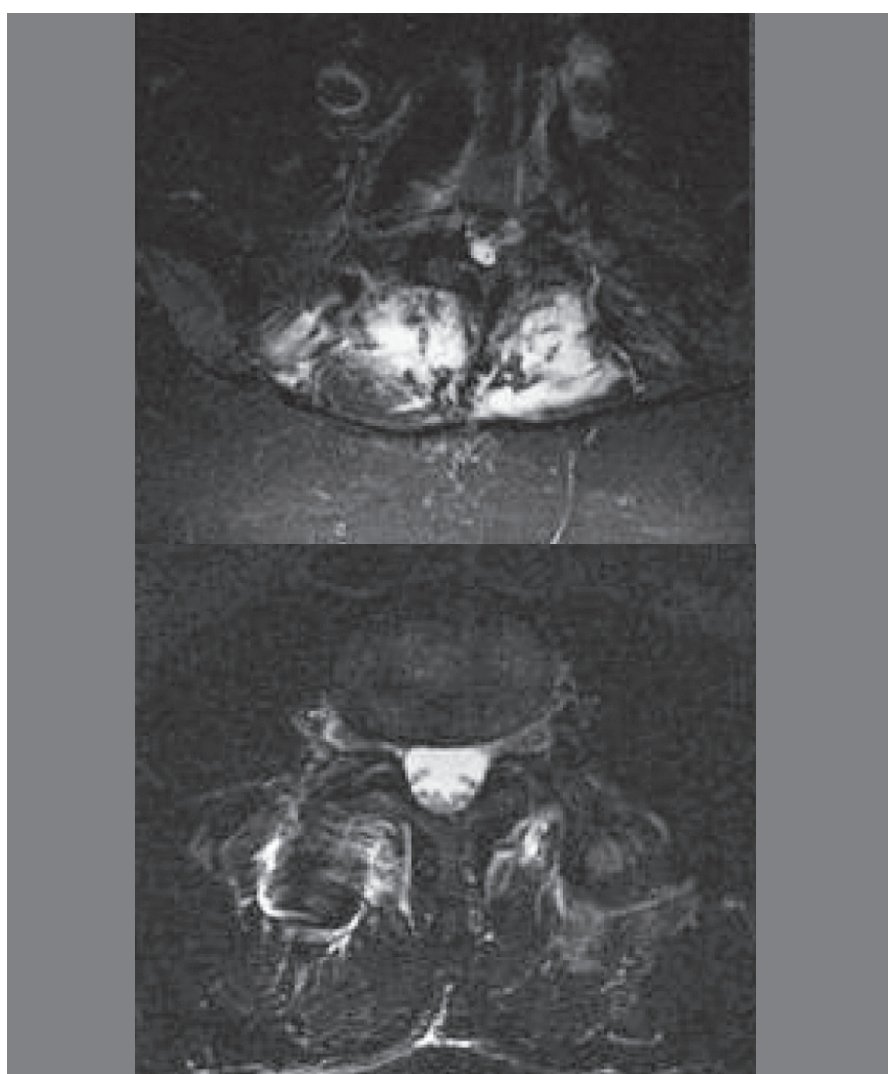

Figura 3. Área do multífido (mm2) (12 meses de pós-operatório).

tendo os mesmos resultados da abordagem convencional aberta ${ }^{5}$. Sua base utiliza-se dos mesmos princípios preconizados na cirurgia aberta, porém sob nova perspectiva, reduzindo a lesão de importantes estruturas estabilizadoras lombares, como o músculo multífido, e limitando o trauma cirúrgico à área de interesse da doença ${ }^{5}$.

Os resultados obtidos neste estudo confirmam que a artrodese lombar minimamente invasiva reduz a lesão muscular iatrogênica e morbidade associada, permitindo ao cirurgião realizar a operação de forma tão eficaz quanto a cirurgia convencional aberta, porém com maior precisão.

Existe lesão dos músculos paraespinhais em ambas as abordagens, com redução da força muscular de extensão do tronco em muitos casos. Entretanto a MIS demonstrou menor grau de lesão muscular do multífido em observações a curto e longo prazo e recuperação da força muscular consideravelmente mais rápida do que na cirurgia aberta ${ }^{5,8,9}$.

Estudos prévios em pacientes operados de hérnia de disco intervertebral evidenciaram anormalidades estruturais do músculo multífido encontradas em eletromiografias e biópsias pós-operatórias $^{1}$. Ocorre uma diminuição da força e atrofia muscular. Achados estes que se encontram relacionados com a clínica apresentada pelos pacientes ${ }^{3}$. Dores lombares e ciáticas persistentes e recorrentes, além de outras deficiências, podem existir após cirurgias lombares. É a chamada Síndrome pós-laminectomia (SPL). Trata-se de um verdadeiro desafio diagnóstico e terapêutico que ocorre em cerca de 10 por cento dos pacientes operados da coluna. Sua origem pode estar relacionada à atrofia por desnervação marcante dos músculos do dorso em seus segmentos lesados, levando à perda de sustentação muscular funcional e posterior aumento de tensão e déficit biomecânico ${ }^{3,8}$

As análise das variáveis idade e sexo já demonstraram não apresentar níveis estatisticamente significativos na comparação entre os tipos de cirurgia em análise prévia ${ }^{10}$.

Aumento na espessura muscular paraespinhal no pós-operatório imediato pode ser devido ao dano intraoperatório e reflete o 
edema intra e extracelular encontrado ${ }^{13}$. A redução da espessura muscular paraespinhal pode ser vista no pós-operatório tardio, tão logo o edema regrida ${ }^{8}$. Os resultados encontrados também estão de acordo com a literatura, mostrando que a MIS diminui o edema pós-operatório em comparação com a cirurgia aberta convencional.

As imagens da RNM de alta resolução são úteis na avaliação sequencial das mudanças pós-operatórias na coluna e demonstram melhor o conteúdo intraespinhal do que outras modalidades de imagem. Implantes modernos com materiais de titânio menos ferromagnéticos e cortes sequenciais permitem uma suscetibilidade aos artefatos magnéticos de forma menos servera ${ }^{11}$.

\section{CONCLUSÃO}

A abordagem minimamente invasiva para artrodese de um nível causa; menor atrofia do multífido, menor grau de degeneração gordurosa e menos edema pós-operatório, quando comparado à cirurgia aberta convencional. Esses achados confirmam que um melhor resultado da cirurgia para fusão lombar pode ser obtido por meio da MIS e estão associados com menor dor lombar e incapacidade funcional, devendo, portanto, ser considerados abordagem preferencial para os pacientes que apresentem indicação de cirurgia.

\section{REFERÊNCIAS}

1. Fan $S, H u Z$, Zhao F, Zhao X, Huang Y, Fang X. Multifidus muscle changes and clinical effects of one-level posterior lumbar interbody fusion: minimally invasive procedure versus conventional open approach. Eur Spine J. 2010;19(2):316-24.

2. Sihvonen T, Herno A, Paljärvi L, Airaksinen O, Partanen J, Tapaninaho A. Local denervation atrophy of paraspinal muscles in postoperative failed back syndrome. Spine (Phila Pa 1976). 1993;18(5):575-81.

3. Hyun SJ, Kim YB, Kim YS, Park SW, Nam TK, Hong HJ, Kwon JT. Postoperative changes in paraspinal muscle volume: comparison between paramedian interfascial and midline approaches for lumbar fusion. J Korean Med Sci. 2007;22(4):646-51.

4. Menezes CM, Ferreira Júnior MA, Falcon RS, Oliveira DA. Artrodese minimamente invasiva para espondilolistese de baixo grau. Coluna/Columna. 2008;7(3):241-45.

5. Kim DY, Lee SH, Chung SK, Lee HY. Comparison of multifidus muscle atrophy and trunk extension muscle strength: percutaneous versus open pedicle screw fixation. Spine (Phila Pa 1976). $2005 ; 30(1): 123-9$.

6. Rutherford EE, Tarplett LJ, Davies EM, Harley JM, King LJ. Lumbar spine fusion and stabilization: hardware, techniques, and imaging appearances. Radiographics. 2007;27(6):1737-49

7. Weber BR, Grob D, Dvorák J, Müntener M. Posterior surgical approach to the lumbar spine and its effect on the multifidus muscle. Spine (Phila Pa 1976). 1997;22(15):1765-72.

8. Wu RH, Fraser JF, Härtl R. Minimal access versus open transforaminal lumbar interbody fusion: meta-analysis of fusion rates. Spine (Phila Pa 1976). 2010;35(26):2273-81.

9. Rantanen J, Hurme M, Falck B, Alaranta H, Nykvist F, Lehto M, Einola S, et al. The lumbar multifidus muscle five years after surgery for a lumbar intervertebral disc herniation. Spine (Phila Pa 1976). 1993;18(5):568-74

10. Kim K, IsuT, Sugawara A, Matsumoto R, Isobe M. Comparison of the effect of 3 different approaches to the lumbar spinal canal on postoperative paraspinal muscle damage. Surg Neurol. 2008;69(2):109-13

11. Mellado JM, Calmet J, Olona M, Esteve C, Camins A, Pérez Del Palomar L, et al. Surgically repaired massive rotator cuff tears: MRI of tendon integrity, muscle fatty degeneration, and muscle atrophy correlated with intraoperative and clinical findings. AJR Am J Roentgenol. 2005;184(5):1456-63.

12. Menezes CM, Falcon RS, Ferreira Júnior MA, Alencar J. Avaliacao clínica radiológica da artrodese lombar transforaminal aberta versus minimamente invasiva. Coluna/Columna. 2009;8(3):297-302.

13. Suwa H, Hanakita J, Ohshita N, Gotoh K, Matsuoka N, Morizane A. Postoperative Chanes in Paraspinal Muscle Thickness After Various Lumbar Back Surgery Procedures. Neurol Med Chir. 2000;40:151-55.

14. Kim K, Isu T, Sugawara A, Matsumoto R, Isobe M. Comparison of the effect of 3 different approaches to the lumbar spinal canal on postoperative paraspinal muscle damage. Surgical Neurology. 2008:69:109-13. 